\title{
Resumen Ejecutivo de CLIVAR-España EL CLIMA EN LA PENÍNSULA IBÉRICA
}

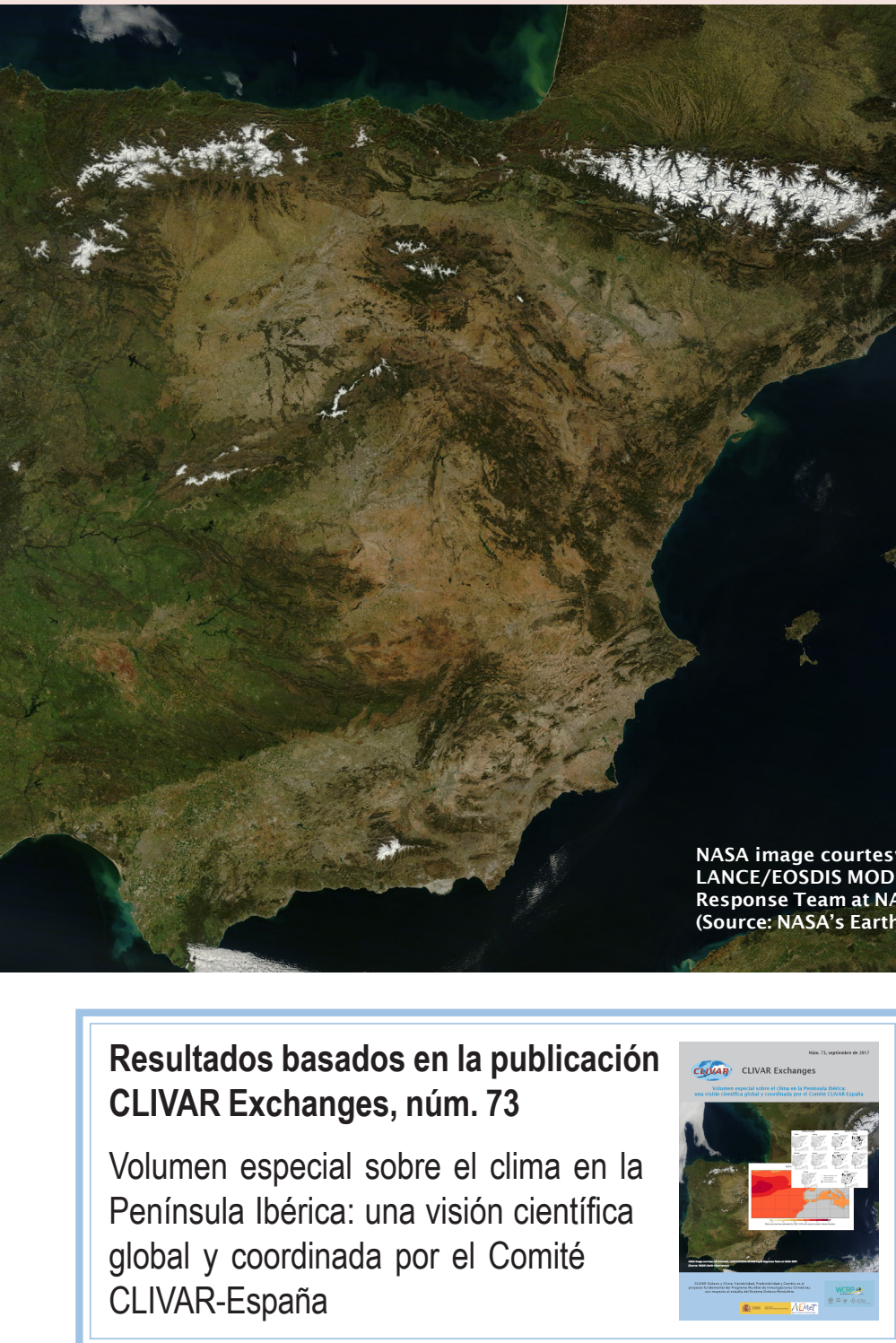




\section{Paleoclima}

El clima de la Península Ibérica de los últimos 2000 años está controlado por las fluctuaciones (y las interacciones) en los modos climáticos (ej. NAO, EA, SCAND), la actividad solar y la actividad volcánica.

La Pequeña Edad del Hielo (1300-1850 AD) fue un periodo climáticamente muy variable a escala temporal y espacial, aunque con predominio de condiciones frías y húmedas y un incremento de eventos extremos en la Península Ibérica.

El clima de la Península Ibérica durante la Anomalía Climática Medieval (900-1300 AD) se caracterizó por condiciones cálidas y sobre todo áridas si bien, localmente, se documenta un aumento en la frecuencia de tormentas.

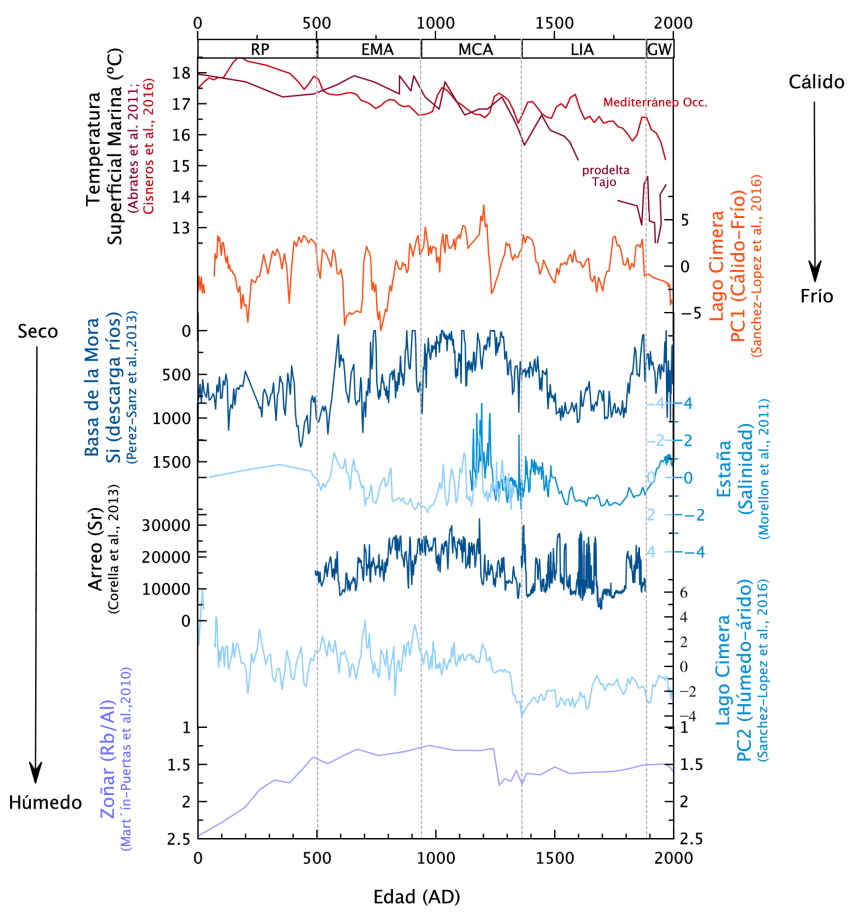

Selección de algunos registros paleoclimáticos en la Península Ibérica, que muestran los cambios de temperatura (arriba, en tonos rojizos) y humedad (abajo, en tonos azulados) en los últimos 2000 años, mediante diversos proxys. 


\section{OBSERVACIONES CLIMÁTICAS}

Se han desarrollado un gran número de bases de datos interpoladas de uso específico, aunque gran parte de ellas no se encuentran fácilmente o son poco accesibles para la comunidad científica o para evaluar impactos.

Faltan análisis sistemáticos de comparación de los productos desarrollados o de la incertidumbre observacional que introducen al ser utilizados como referencia en otros análisis.

La homogeneización de series mensuales ha alcanzado suficiente madurez y fiabilidad y se establece como proceso previo al abordar el análisis de series observacionales. Se está avanzando mucho en la homogeneización de series diarias necesarias para estudiar la variabilidad de los valores extremos.

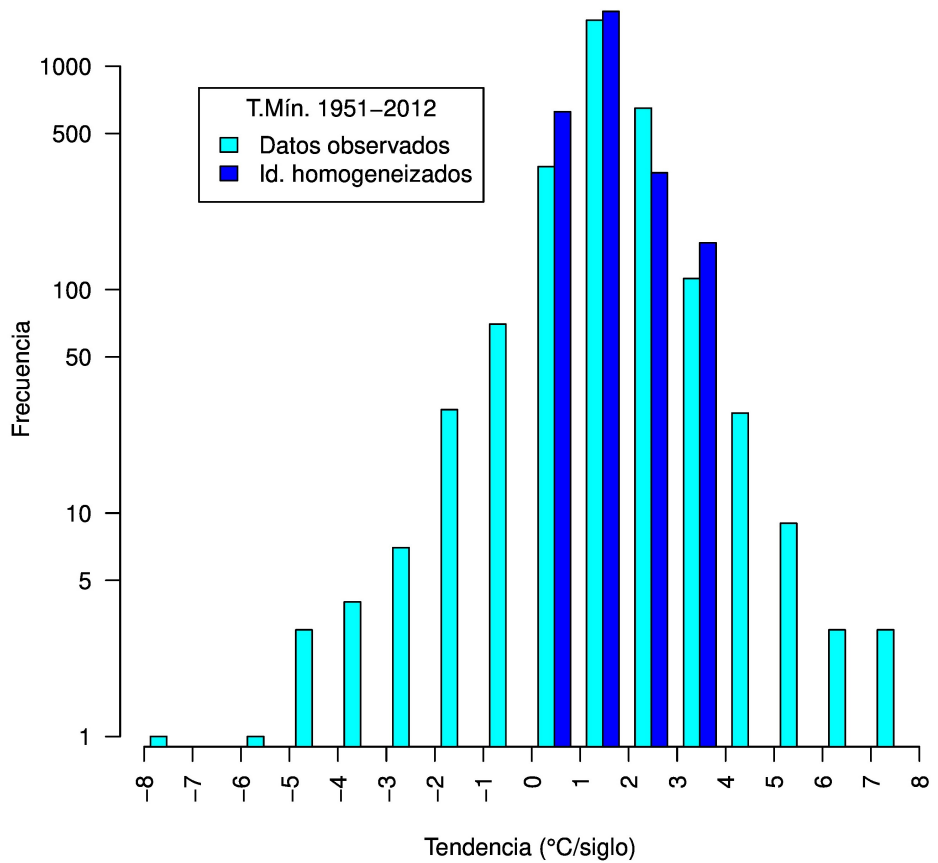

Tendencias de las temperaturas mínimas del aire en España antes y después de la homogeneización de las series. Las inhomogeneidades de los datos brutos de observación dan como resultado una dispersión anormalmente alta de las tendencias calculadas. 


\section{TEndencias}

Existe un claro incremento de temperatura del aire en España durante las últimas décadas.

No se han producido cambios notables en la velocidad del viento (media confianza, alto consenso), pero sí en los niveles de humedad relativa, que han descendido de forma generalizada en las últimas décadas.

Las tendencias en precipitación no muestran grandes cambios a nivel general, pero presentan una elevada variabilidad estacional y espacial.

Los extremos térmicos se han incrementado, sobre todo con el aumento de las olas de calor (elevada confianza, alto consenso), en cambio, no se asiste a un aumento generalizado de los extremos pluviométricos.

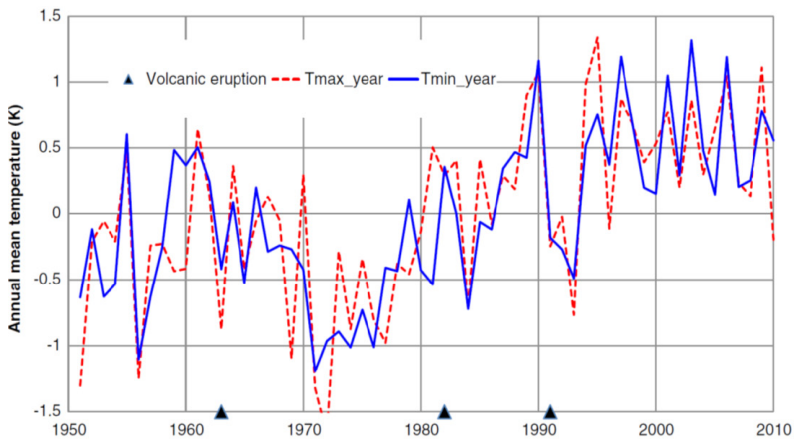

Media anual de las temperaturas máximas y mínimas (1951-2010). Los valores se expresan en anomalías respecto al periodo $1950-2010$.

\section{VARIABILIDAD}

La combinación de la NAO con otros patrones de teleconexión explican la variabilidad climática de la región. No obstante hay que considerar que las relaciones no son estacionarias.

Los ejercicios de predicción estacional y decadal muestran un gran potencial en Europa: la temperatura de la superficie del océano, el vórtice polar o la extensión de hielo marino son potenciales predictores.

Las proyecciones regionales confirman que el Mediterráneo es un punto caliente de cambio climático. No obstante, existen considerables incertidumbres sobre los cambios en circulación atmosférica y oceánica. 


\section{Observaciones en el oceÁno}

Se ha realizado un gran esfuerzo en monitorizar regularmente zonas marinas alrededor de la Península Ibérica.

La extrema pérdida de calor en el invierno 2004/2005 afectó la formación de masas de agua en la región, alterando características y tendencias climáticas en aguas profundas e intermedias del Atlántico Nordeste y el Mediterráneo Occidental durante esta última década. Estas alteraciones contribuyeron directamente al denominado hiato del calentamiento global.

Gracias a mejores estimaciones de movimientos verticales de la corteza terrestre y una mayor cobertura de altimetría por satélite se ha mejorado la precisión en las medidas del nivel del mar. Los resultados confirman unas subidas anuales sostenidas de 1 a $4 \mathrm{~mm}$ en el Atlántico y de 2,5 a $3 \mathrm{~mm}$ en el Mediterráneo.

\section{Regionalización. Clima presente}

Regionalizar los modelos permite disponer de información a mayor resolución. Al representar mejor las características de la superficie y la orografía mejoran la distribución espacial regional y local de variables climáticas para evaluar proyecciones climáticas frente a observaciones y medir su confianza respecto a los modelos forzantes originales.

Es altamente recomendable evaluar diversas variables para cada proceso, no solo temperatura y precipitación, pues permite identificar y evitar posibles compensaciones de errores.

La regionalización lleva incertidumbres inherentes a cambios de modelo, dinámico o estadístico, que se exploran utilizando conjuntos de simulaciones cuyo análisis permite establecer señales más robustas.

Actualmente se está incorporando más complejidad, ya sea a través de usar técnicas más complejas en los estadísticos o bien ampliando componentes del sistema climático a escala regional: océano, química, etc. 


\section{Regionalización. Clima futuro}

La mayor fuente de incertidumbre para el futuro cercano (hasta 2050) está en los modelos globales. Los regionales preservan las tendencias de los modelos globales moderando los cambios más extremos.

En los modelos regionales para 2021-2050, los mayores cambios con respecto a 1971-2000 se proyectan en verano, con incrementos de temperatura entre 1,1 y $2,8{ }^{\circ} \mathrm{C}$, y descensos de hasta el $30 \%$ en precipitación, independientemente del escenario de emisiones.

Otros cambios significativos en este periodo son: disminución de días de nieve y de viento, y aumento de periodos de sequía.

En AdapteCCa (http://escenarios.adaptecca.es) se muestran las proyecciones más recientes para España peninsular y Baleares.

\begin{tabular}{|c|c|c|c|c|c|c|c|c|c|c|c|c|c|c|c|c|c|}
\hline \multirow[b]{3}{*}{ (6) MGCs-RCP85 - } & \multicolumn{16}{|c|}{ Temperatura $\left({ }^{\circ} \mathrm{C}\right)$} & \\
\hline & \multicolumn{4}{|c|}{ DEF } & \multicolumn{4}{|c|}{ MAM } & \multicolumn{4}{|c|}{ JJA } & \multicolumn{4}{|c|}{ SON } & \\
\hline & 1.0 & 1.2 & 1.4 & 1.5 & 0.9 & 1.1 & 1.4 & 1.6 & 1.4 & 2.1 & 2.3 & 2.9 & 1.2 & 1.3 & 32.2 & 2.6 & \\
\hline (12) MREs-RCP85 - & 0.9 & 1.1 & 1.4 & 1.5 & 1.0 & 1.1 & 1.5 & 1.7 & 1.1 & 1.4 & 2.3 & 2.8 & 1.2 & 1.3 & 32.3 & 32.7 & \\
\hline (16) MRCs-RCP85 - & 0.6 & 0.8 & 1.2 & 1.4 & 0.8 & 0.9 & 1.3 & 1.4 & 1.1 & 1.2 & 2.0 & 2.4 & 1.1 & 1.2 & 21.9 & 2.4 & 2 \\
\hline (6) MGCs-RCP45 - & 1.0 & 1.1 & 1.2 & 1.2 & 0.6 & 0.9 & 1.3 & 1.3 & 1.4 & 1.7 & 2.1 & 2.6 & 1.0 & 1.2 & 2.0 & 2.3 & -1 \\
\hline (12) MREs-RCP45 - & 0.9 & 1.0 & 1.2 & 1.3 & 0.6 & 0.8 & 1.3 & 1.4 & 1.1 & 1.3 & 1.9 & 2.5 & 1.0 & 1.2 & 2.1 & 2.4 & -0 \\
\hline (15) MRCs-RCP45 - & 0.7 & 0.9 & 1.1 & 1.2 & 0.3 & 0.5 & 1.2 & 1.4 & 1.3 & 1.3 & 1.8 & 2.4 & 1.0 & 1.2 & 2.2 & 2.3 & - \\
\hline (12) MGCs-All - & 0.9 & 1.1 & 1.3 & 1.5 & 0.7 & 1.0 & 1.4 & 1.6 & 1.3 & 1.8 & 2.3 & 2.9 & 1.1 & 1.2 & 2.1 & 2.5 & \\
\hline (24) MREs-All - & 0.9 & 1.0 & 1.3 & 1.5 & 0.7 & 1.1 & 1.4 & 1.6 & 1.1 & 1.3 & 2.2 & 2.8 & 1.0 & 1.3 & $\begin{array}{ll}3 & 2.2\end{array}$ & 2.6 & \\
\hline \multirow[t]{5}{*}{ (31) MRCs-All } & 0.6 & 0.8 & 1.2 & 1.3 & 0.3 & 0.8 & 1.3 & 1.4 & 1.2 & 1.3 & 2.0 & 2.4 & 1.0 & 1.2 & 2.2 & 2.4 & \\
\hline & $\Gamma$ & $T$ & $T$ & $T$ & $T$ & $T$ & $T$ & $T$ & & & & & $T$ & $T$ & 1 & 7 & \\
\hline & 5 & 25 & 75 & 95 & 5 & 25 & 75 & 95 & 5 & 25 & 75 & 95 & 5 & 25 & $5 \quad 75$ & 95 & \\
\hline & \multicolumn{16}{|c|}{ Precipitación (\%) } & \\
\hline & \multicolumn{4}{|c|}{ DEF } & \multicolumn{4}{|c|}{ MAM } & \multicolumn{4}{|c|}{ JJA } & \multicolumn{4}{|c|}{ SON } & \\
\hline (6) MGCs-RCP85 & -12 & -5 & 2 & 4 & -18 & -12 & -5 & 4 & -23 & -21 & -6 & -4 & -13 & -12 & -5 & -1 & -40 \\
\hline (12) MREs-RCP85 - & -14 & -9 & -3 & -1 & -17 & -14 & -5 & 5 & -19 & -14 & -5 & -0 & -21 & -15 & -8 & -6 & \\
\hline (16) MRCs-RCP85 - & -6 & -3 & 1 & 2 & -22 & -16 & -7 & -5 & -30 & -24 & -2 & -0 & -16 & -15 & 5 & 7 & -20 \\
\hline (6) MGCs-RCP45 - & -7 & -5 & 1 & 9 & -19 & -11 & 2 & 5 & -22 & -18 & -7 & -2 & -19 & -17 & -10 & -7 & \\
\hline (12) MREs-RCP45 - & -12 & -9 & -1 & 11 & -21 & -15 & 1 & 3 & -23 & -16 & -8 & -5 & -21 & -18 & -9 & -5 & -0 \\
\hline (15) MRCs-RCP45 - & -4 & -3 & 4 & 5 & -21 & -18 & -7 & -3 & -29 & -19 & -3 & 1 & -20 & -18 & -11 & -9 & \\
\hline (12) MGCs-All - & -11 & -6 & 2 & 7 & -20 & -14 & 2 & 6 & -23 & -19 & -6 & -3 & -18 & -14 & -7 & -2 & --20 \\
\hline (24) MREs-All - & -13 & -9 & -3 & 9 & -19 & -15 & 1 & 4 & -22 & -14 & -7 & -3 & -22 & -18 & -8 & -4 & \\
\hline (31) MRCs-All & -5 & -3 & 2 & 5 & -22 & -17 & -7 & -3 & -30 & -23 & -3 & 0 & -19 & -15 & -1 & 6 & -40 \\
\hline & $\Gamma$ & 1 & $T$ & 1 & $T$ & 1 & $T$ & 1 & $T$ & $T$ & 1 & $T$ & $T$ & 1 & $T$ & $\neg$ & \\
\hline & 5 & 25 & 75 & 95 & 5 & 25 & 75 & 95 & 5 & 25 & 75 & 95 & 5 & 25 & 75 & 95 & \\
\hline
\end{tabular}

Proyecciones para 2021-2050, respecto al promedio de 1971-2000, según dos escenarios de emisiones (RCP8.5, RCP 4.5 y ambos «All») para España continentaly Baleares. Cambios medios de temperatura en superficie $\left({ }^{\circ} \mathrm{C}\right.$, arriba) y precipitación (\%, abajo),por estaciones del año, según las proyecciones de los MCG, MRE o MRC (en filas diferentes

para cada escenario). En columnas se dan los percentiles (5, 25, 75 y 95) para cada caso. El rango 25-75 se utiliza para mostrar la dispersión, y los percentiles 5 y 95 caracterizan los extremos. 


\section{Proyecciones en el océano}

Se espera que la temperatura superficial del mar alrededor de las costas españolas aumente entre 2 y $3^{\circ} \mathrm{C}$ a final de siglo.

El nivel del mar podría subir hasta $80-100 \mathrm{~cm}$, lo cual tendría consecuencias graves para el entorno costero en muchas zonas.

Los valores medios de altura de ola experimentarán una ligera disminución (5-10 \%) tanto en el Mediterráneo como en el Atlántico.
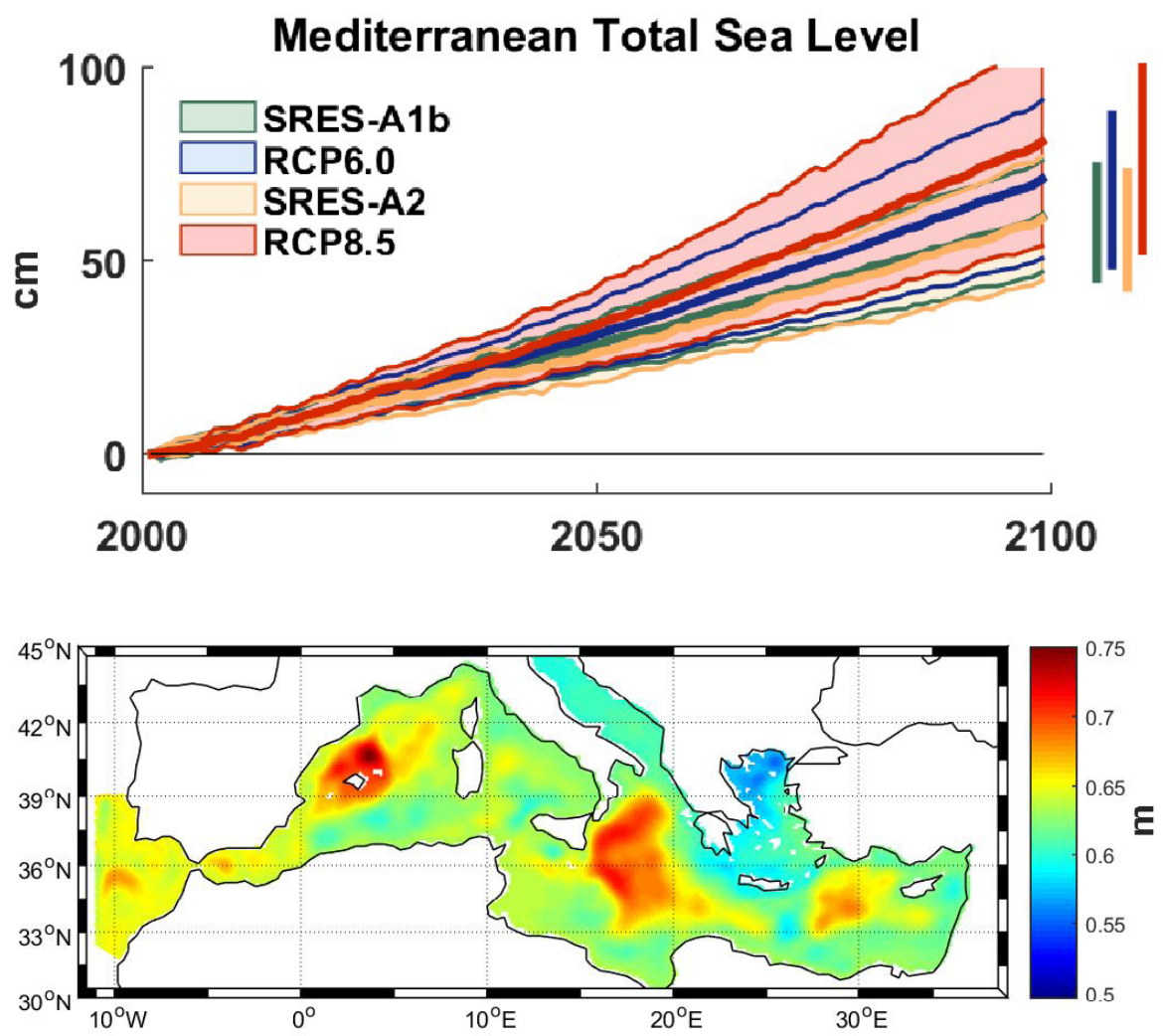

Arriba, proyecciones del valor promedio para toda la cuenca del nivel del mar Mediterráneo (en cm).

Abajo, distribución espacial de la proyección de nivel del mar Mediterráneo (en $\mathrm{m}$ ) bajo un escenario moderado (RCP4.5 o A1b). 
Progresos en la detección y proyecciones de cambio climático en España desde el informe 2010 de evaluación regional del clima pasado, presente y futuro del Comité CLIVAR-España,

por Enrique Sánchez, Belén Rodríguez-Fonseca, lleana Bladé, Manola Brunet, Roland Aznar, Isabel Cacho,

María Jesús Casado, Luis Gimeno, José Manuel Gutiérrez, Gabriel Jordá, Alicia Lavín, José Antonio López, Jordi Salat y Blas Valero

Una breve síntesis de la evolución climática de la península ibérica durante los últimos 2000 años por S. Giralt, A. Moreno, I. Cacho y B. Valero-Garcés

Observaciones climáticas atmosféricas y reconstrucciones instrumentales sobre la península ibérica I. Obtención de series climáticas de alta calidad

por J. A. Guijarro, C. Azorín-Molina, J. C. González-Hidalgo, A. Sánchez-Lorenzo, S. Herrera y J. A. López

Observaciones climáticas y reconstrucción instrumental.

II. Desarrollo de productos climáticos interpolados de alta calidad

por S. Herrera, J. J. Miró Pérez, P. Quintana-Seguí, J. Gonzalo, J. A. Ruiz-Arias, J. C. González-Hidalgo, J. A. Guijarro y J. A. López

Tendencias recientes de las variables atmosféricas en España por S. M. Vicente Serrano y E. Rodríguez Camino

Modos de variabilidad que afectan al suroeste de Europa por B. Rodríguez-Fonseca, M. J. Casado y D. Barriopedro

Variabilidad oceánica y cambios de nivel del mar alrededor de

la península ibérica, Baleares y Canarias

por J. Salat, A. Lavín, C. González-Pola, P. Vélez-Belchí, R. Sánchez, M. Vargas-Yáñez, J. García-Lafuente, M. Marcos y D. Gomis

Proyecciones de clima regional sobre España: atmósfera. Evaluación del clima presente por J. P. Montávez, J. Fernández, A. Casanueva, J. M. Gutiérrez y E. Sánchez

Proyecciones de clima regional sobre España: atmósfera. Proyecciones de clima futuro por J. Fernández, A. Casanueva, J. P. Montávez, M. Á. Gaertner, M. J. Casado, R. Manzanas y J. M. Gutiérrez

Proyecciones de clima regional marino sobre España por G. Jordà, M. Menéndez, R. Aznar y A. Sánchez-Arcilla

\section{Comité Nacional CLIVAR-España}

Su principal objetivo es promover y coordinar la contribución española al programa CLIVAR (Climate Variability and Predictability) con los objetivos científicos, entre otros, de describir y comprender los procesos físicos responsables de la variabilidad climática, extender el registro de variabilidad climática a lo largo de las escalas de interés, extender el rango y la fiabilidad de las predicciones climáticas, y comprender y predecir la respuesta del sistema climático al aumento en la concentración de gases de efecto invernadero y aerosoles.

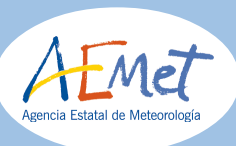

www.aemet.es
CLIVAR Océano y Clima:

Variabilidad, Predictibilidad y Cambio es el proyecto fundamental del

Programa Mundial de Investigaciones

Climáticas con respecto al estudio del Sistema Océano-Atmósfera

C AEMET (Ministerio para la Transición Ecológica)

(c) CLIVAR-España

Madrid, 2019
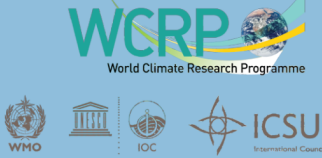

NIPO: 639-19-001-4

https://doi.org/10.31978/639-19-001-4

Depósito Legal: M-23222-2019

Imprime: Nemac Comunicación SL 